\title{
Electrostatic interactions in periodic Coulomb and dipolar systems
}

\author{
B. Cichocki, ${ }^{*}$ B. U. Felderhof, and K. Hinsen \\ Institut für Theoretische Physik A, Rheinisch-Westfälische Technische Hochschule Aachen, Templergraben 55, \\ 5100 Aachen, Federal Republic of Germany
}

(Received 7 December 1988)

\begin{abstract}
We present a new method for calculating electrostatic interactions in periodic systems with charged or dipolar particles. The method is appreciably faster than the Ewald summation method used until now in computer simulations of ionic and dipolar systems. The method may also be used for calculating the periodic crystal field in solids.
\end{abstract}

\section{INTRODUCTION}

For systems of charged particles, such as ionic solutions, plasmas, and fused salts, and for systems of dipolar particles, such as polar liquids, the long range of the interactions causes severe problems. In computer simulations one usually prefers to use periodic boundary conditions, and this is difficult to combine with the long-range Coulomb or dipolar interaction. ${ }^{1-3}$ Some attempts have been made to simulate systems in finite geometry, ${ }^{4}$ but it must be feared that in such situations surface effects are difficult to overcome. In simulations with periodic boundary conditions several methods have been employed, the main ones being spherical truncation of the interaction, possibly in combination with a reaction field, ${ }^{5}$ and the method of Ewald summation. ${ }^{6,7}$ In this article we propose a new scheme which in our opinion supersedes existing methods.

A prime condition for a successful simulation of systems with long-range interactions is a clear physical picture which permits a Hamiltonian formulation. Maxwell theory allows a transparent description on the macroscopic length scale of large, but finite samples. Thus we follow earlier work by de Leeuw et $a l^{7}{ }^{7}$ and by Felderhof ${ }^{8}$ in which periodic systems of finite extent are considered and Maxwell theory is used for a calculation of the macroscopic field which pervades the system in addition to the periodic microscopic field. Such an approach allows a simple derivation of macroscopic fluctuation theorems and opens the way for a proper statistical-mechanical treatment, including a discussion of the thermodynamic limit. ${ }^{9}$

Reaction field methods have also met with some success, ${ }^{10,11}$ but the statistical-mechanical treatment and macroscopic considerations are less straightforward. The inherent difficulties have been discussed in some detail by de Leeuw et $a l .^{2}$ In our view the method of finite periodic systems is to be preferred.

Ladd $^{12}$ has proposed a method for evaluating the electrostatic energy of a periodic polar system by use of a multipole expansion of the interaction of a molecule with those outside its cell of nearest images. He investigated the lowest-order contributions to the potential energy.

In a large, but finite sample of periodically repeated cells the electrostatic potential is well approximated by the sum of a macroscopic Maxwell potential, calculated from Maxwell's equations, and a periodic crystal potential. If the shape of the sample is ellipsoidal, then the macroscopic Maxwell field is uniform and linear in the applied field and the polarization. The periodic crystal potential may be constructed as a sum of Wigner potentials, the latter being defined as the electrostatic potential of a Wigner crystal consisting of a periodic cubic array of identical point charges embedded in a neutralizing background. ${ }^{13}$ We show that the electrostatic interactions between particles may be expressed in terms of the Wigner potential.

We present a method by which the Wigner potential may be calculated quickly and accurately. The calculation is based on an expansion of the potential in harmonic polynomials about suitably chosen points in the unit cell of a simple cubic lattice. The coefficients of the expansions may be evaluated once and for all by Ewald summation. A diskette with numerical values of the coefficients is available from the authors upon request. Together with the theory presented here this should allow easy formulation of a computer algorithm. We have tested the method in a calculation of the dielectric constant of a nonpolar liquid. The results will be published elsewhere.

In the following we assume a simple cubic lattice, as is usually employed in computer simulations. The theory may be extended to general Bravais lattices and provides a method for calculating the crystal potential in solids.

\section{IONIC CRYSTALS OF FINITE SIZE}

We consider a cube with sides $L$ and volume $V=L^{3}$ centered at the origin. The cube contains $N$ charges $e_{1}, \ldots, e_{N}$ situated at positions $\mathbf{R}_{1}, \ldots, \mathbf{R}_{N}$. We assume charge neutrality

$$
\sum_{j=1}^{N} e_{j}=0 .
$$

Hence the total dipole moment

$$
\mathbf{M}=\sum_{j=1}^{N} e_{j} \mathbf{R}_{j}
$$

is independent of the choice of origin. As a consequence, if the cube is located in vacuum, the electrostatic potential $\phi_{V}(\mathbf{r})$, which is the solution of Poisson's equation 


$$
\nabla^{2} \phi_{V}=-4 \pi \sum_{j=1}^{N} e_{j} \delta\left(\mathbf{r}-\mathbf{R}_{j}\right),
$$

has asymptotic behavior

$$
\phi_{V}(\mathbf{r}) \approx \frac{\mathbf{M} \cdot \mathbf{r}}{r^{3}} \text { for } r \gg L .
$$

Next we consider simple cubic crystals built out of periodically repeated unit cells as described above. For any large simply connected volume $\Omega$ we may find the crystal of maximum size which just fits inside $\Omega$. This single crystal consists of $\mathcal{N}(\Omega, L)$ elementary cubes. The total number of charges is $\mathcal{N N}$ and the system has total dipole moment $\boldsymbol{M}=\mathcal{N M}$. The origin is chosen to be in the center of the central cube. We denote the central cube as $C(L)$. For $r \gg \mathcal{N} L$ the electrostatic potential $\phi(\mathbf{r})$ has the behavior (2.4) with $\mathbf{M}$ replaced by the total dipole moment $\boldsymbol{M}$. We denote the crenellated single crystal constructed above as $C(\Omega, L)$.

For the sake of generality we replace the vacuum outside $\Omega$ by a uniform medium with dielectric constant $\epsilon^{\prime}$. We denote the complete geometry obtained in this manner as $C\left(\Omega, L, \epsilon^{\prime}\right)$. At asymptotic distance the potential is a reduced dipole potential. The electrostatic potential inside $\Omega$ depends on the detailed positions of the charges and is influenced by image or reaction field effects.

In the case where $\Omega$ is a sphere the electrostatic potential $\phi\left(\mathbf{r}, \epsilon^{\prime}\right)$ may be found exactly everywhere in space by use of the image method. However, this knowledge is of no practical use when $\Omega$ and $\mathcal{N}$ are large. In general, for sufficiently large bodies the potential inside $\Omega$ is well approximated by the sum of a macroscopic Maxwell potential $\phi_{M}\left(\mathbf{r}, \Omega, \epsilon^{\prime}\right)$ and a periodic crystal potential $\phi_{c}(\mathbf{r}, L)$,

$$
\phi\left(\mathbf{r}, \Omega, L, \epsilon^{\prime}\right) \approx \phi_{M}\left(\mathbf{r}, \Omega, \epsilon^{\prime}\right)+\phi_{c}(\mathbf{r}, L), \quad r \in \Omega .
$$

From a macroscopic point of view, i.e., when averaging over a length scale large compared with $L$, we deal with a neutral, uniformly polarized body with dielectric constant 1 surrounded by a medium with dielectric constant $\epsilon^{\prime}$. The uniform polarization is given by $\mathbf{P}=\mathbf{M} / V$. The Maxwell potential $\phi_{M}\left(\mathbf{r}, \Omega, \epsilon^{\prime}\right)$ is defined as the solution of Maxwell's equations of electrostatics for this macroscopic geometry. The crystal potential $\phi_{c}(\mathbf{r}, L)$ has the periodicity of the lattice and accounts for the detailed positions of the charges. We allow a uniform applied electric field $\mathbf{E}_{0}$ which is included in the Maxwell potential. Hence at large distances from $\Omega$ the Maxwell field is given by

$$
\phi_{M}\left(\mathbf{r}, \Omega, \epsilon^{\prime}\right) \approx-\mathbf{E}_{0} \cdot \mathbf{r}+\frac{M^{\prime} \cdot \mathbf{r}}{\epsilon^{\prime} r^{3}},
$$

where $\mathcal{M}^{\prime}$ is the external dipole moment. Outside $\Omega$ the crystal potential $\phi_{c}$ continues to a constant which is not relevant for our purposes and which we leave undetermined. In the thermodynamic limit $\Omega \rightarrow \infty$ the relation (2.5) yields the exact potential in the bulk of the body. Clearly, the limiting potential depends on sample shape via the Maxwell term.

We consider in particular an ellipsoidal volume $\Omega$. In that case the Maxwell potential $\phi_{M}\left(r, \Omega, \epsilon^{\prime}\right)$ may be found exactly. We choose coordinate axes along the principal axes of the ellipsoid. The Maxwell field inside the ellipsoid is spatially uniform and has components ${ }^{14}$

$$
E_{M \alpha}\left(\Omega, \epsilon^{\prime}\right)=\frac{\epsilon^{\prime} E_{0 \alpha}-4 \pi n^{(\alpha)} P_{\alpha}}{\epsilon^{\prime}+\left(1-\epsilon^{\prime}\right) n^{(\alpha)}} \quad(\alpha=x, y, z),
$$

where $n^{(x)}, n^{(y)}, n^{(z)}$ are the depolarization coefficients of the ellipsoid. For a sphere $n^{(x)}=n^{(y)}=n^{(z)}=\frac{1}{3}$. The Maxwell potential inside the ellipsoid is given by

$$
\phi_{M}\left(\mathbf{r}, \Omega, \epsilon^{\prime}\right)=-\mathbf{E}_{M}\left(\Omega, \epsilon^{\prime}\right) \cdot \mathbf{r}, \quad r \in \Omega .
$$

The considerations of this section may be extended to crystals built out of unit cells which are not cubic. Any Bravais lattice with basis vectors $\mathbf{a}_{1}, \mathbf{a}_{2}, \mathbf{a}_{3}$ may be used. In the sequel we restrict ourselves to cubic cells.

\section{CRYSTAL POTENTIAL}

In this section we study the crystal potential $\phi_{c}(\mathbf{r}, L)$ introduced in (2.5) in more detail. By definition $\phi_{c}(r, L)$ is a solution of Poisson's equation which is periodic in the infinite lattice of cubic cells. In the basic unit cell $\phi_{c}(\mathbf{r}, L)$ satisfies

$$
\nabla^{2} \phi_{c}(\mathbf{r}, L)=-4 \pi \sum_{j=1}^{N} e_{j} \delta\left(\mathbf{r}-\mathbf{R}_{j}\right), \quad \mathbf{r} \in C(L) .
$$

On account of periodicity the crystal potential satisfies the boundary condition $\boldsymbol{v} \cdot \nabla \phi_{c}=0$ at the cell surface, where $v$ is the normal to the boundary. Clearly, Poisson's equation (3.1) and the periodic boundary conditions determine $\phi_{c}(\mathbf{r}, L)$ up to an additive constant.

We may find an explicit expression for the crystal potential $\phi_{c}(\mathbf{r}, L)$ by relating it to the potential of a Wigner solid. ${ }^{12}$ Thus we consider a simple cubic lattice with a charge $e$ at the center of each cube and with a neutralizing uniform background with charge density $\tau=-e / V$. The cells may be indicated by the lattice vectors $\mathbf{n}$, where $n_{x}, n_{y}, n_{z}$ are integers. The charges are at positions $\mathbf{n} L$. The basic cube $C(L)$ has the lattice point $\mathbf{n}=0$ at its center. By definition the Wigner potential is periodic throughout the lattice. In the basic cube it is given by ${ }^{15,16}$

$$
\begin{aligned}
\phi_{W}(\mathbf{r}, L)=e\left[\frac{1}{r}+\right. & \frac{2 \pi}{3} L^{-3} r^{2} \\
+\sum_{\substack{l>0 \\
m}} L^{-l-1}\left(\frac{4 \pi}{2 l+1}\right]^{1 / 2} & \\
& \left.\quad \times A_{l m} r^{l} Y_{l m}(\theta, \varphi)\right], \quad \mathrm{r} \in C(L)
\end{aligned}
$$

where the second term arises from the neutralizing background, and where $Y_{I m}(\theta, \varphi)$ is a spherical harmonic. The coefficients $A_{l m}$ must be such that the potential has the lattice symmetry. As a consequence only even values of $l$ occur and the azimuthal quantum number $m$ must be a multiple of four. Furthermore $A_{20}=0$. The appropriate linear combinations are the fully symmetric kubic harmonics $K_{l \alpha}(\mathbf{r})$ first introduced by Von der Lage and 
Bethe. ${ }^{17}$ In addition, the coefficients must be chosen such that $\boldsymbol{v} \cdot \nabla \phi_{W}=0$ at the cell boundary. Nijboer and de Wette ${ }^{18}$ have derived rapidly converging lattice sums for the coefficients $A_{l m}$.

By the requirement of periodicity the Wigner potential is determined up to a constant and we have made a definite choice in (3.2). Alternatively the potential may be defined as a sum of Coulomb potentials from point charges $e$ placed at the lattice positions. Different values for the constant have been obtained depending on how the conditionally convergent lattice sum is performed. A detailed discussion of this point has been given by de Wette, ${ }^{19}$ Ihm and Cohen, ${ }^{20}$ and recently by Nijboer and Ruijgrok. $^{21}$ The constant appears naturally in the calculation of the electrostatic energy of a Wigner solid. Of course, the energy has a unique value.

We write the Wigner potential in the form

$$
\phi_{W}(\mathbf{r}, L)=\frac{e}{L} w(\mathbf{r} / L),
$$

where the dimensionless potential $w(\mathbf{x})$ is defined for a simple cubic lattice with lattice distance unity. In later sections we shall make a detailed study of this basic function.

The crystal potential $\phi_{c}(\mathbf{r}, L)$ may now be written

$$
\phi_{c}(\mathbf{r}, L)=\frac{1}{L} \sum_{j=1}^{N} e_{j} w\left(\left(\mathbf{r}-\mathbf{R}_{j}\right) / L\right) .
$$

This potential is clearly periodic. Note that an additive constant proportional to $e$ in (3.2) would drop out in (3.4) on account of charge neutrality. The potential satisfies the Poisson equation (3.1), since $\sum_{j} \tau_{j}=0$.

de Wette and Nijboer ${ }^{15}$ have proposed a different expression for the lattice potential. However, in their expression new lattice sums must be evaluated dependent on the positions of the charges in the unit cell. The advantage of $(3.4)$ is that only the basic function $w(\mathbf{x})$ is required.

\section{ELECTROSTATIC ENERGY}

In this section we consider the electrostatic energy for the geometry $C\left(\Omega, L, \epsilon^{\prime}\right)$ in the case where $\Omega$ is an ellipsoid. We approximate the potential by the expression (2.5). The Maxwell potential, given by (2.8), may be written as a sum of two terms

$$
\phi_{M}\left(\mathrm{r}, \Omega, \epsilon^{\prime}\right)=\phi_{M}^{0}\left(\mathrm{r}, \Omega, \epsilon^{\prime}\right)+\phi_{M}^{P}\left(\mathbf{r}, \Omega, \epsilon^{\prime}\right)
$$

corresponding to the decomposition (2.7). We define the crystal potential acting on charge $j$ in the basic cell by

$$
\phi_{c j}^{\prime}(\mathbf{r}, L)=\phi_{c}(\mathbf{r}, L)-\frac{e_{j}}{\left|\mathbf{r}-\mathbf{R}_{j}\right|} .
$$

Here we have subtracted the self-term. The electrostatic energy of the whole system is then given by

$$
\begin{aligned}
U_{\mathcal{N}}\left(\Omega, L, \epsilon^{\prime}\right)=\sum_{\mathrm{n} \in C(\Omega, L)} \sum_{j=1}^{N} e_{j} & {\left[\frac{1}{2} \phi_{c j}^{\prime}\left(\mathbf{R}_{j}, L\right)\right.} \\
& +\frac{1}{2} \phi_{M}^{P}\left(\mathbf{R}_{\mathbf{n} j}, \Omega, \epsilon^{\prime}\right) \\
& \left.+\phi_{M}^{0}\left(\mathbf{R}_{\mathbf{n} j}, \Omega, \epsilon^{\prime}\right)\right],
\end{aligned}
$$

where $\mathbf{R}_{\mathbf{n} j}$ is the position of charge $j$ in cell $\mathbf{n}$. By use of (2.2) and (2.8) we find that there is an equal contribution from each cubic cell, so that

$$
U_{\mathcal{N}}\left(\Omega, L, \epsilon^{\prime}\right)=\mathcal{N} U\left(\Omega, L, \epsilon^{\prime}\right),
$$

with an energy per cell

$$
\begin{aligned}
U\left(\Omega, L, \epsilon^{\prime}\right)= & \frac{1}{2} \sum_{j=1}^{N} e_{j} \phi_{c j}^{\prime}\left(\mathbf{R}_{j}, L\right)-\frac{1}{2} \mathbf{M} \cdot \mathbf{E}_{M}^{P}\left(\Omega, \epsilon^{\prime}\right) \\
& -\mathbf{M} \cdot \mathbf{E}_{M}^{0}\left(\Omega, \epsilon^{\prime}\right) .
\end{aligned}
$$

It follows from (2.7) that the second term is quadratic in the dipole moment $\mathbf{M}$, whereas the third term is linear.

In particular, for the case of a sphere we put $\Omega=S$ and obtain for the two contributions in (2.7)

$$
\begin{aligned}
& \mathbf{E}_{M}^{0}\left(S, \epsilon^{\prime}\right)=\frac{3 \epsilon^{\prime}}{2 \epsilon^{\prime}+1} \mathbf{E}_{0}, \\
& \mathbf{E}_{M}^{P}\left(S, \epsilon^{\prime}\right)=-\frac{4 \pi}{2 \epsilon^{\prime}+1} \frac{\mathbf{M}}{V} .
\end{aligned}
$$

In that case the energy per cell is given by

$$
\begin{aligned}
U\left(S, L, \epsilon^{\prime}\right)= & \frac{1}{2} \sum_{j=1}^{N} e_{j} \phi_{c j}^{\prime}\left(\mathbf{R}_{j}, L\right)+\frac{2 \pi}{2 \epsilon^{\prime}+1} \frac{M^{2}}{V} \\
& -\frac{3 \epsilon^{\prime}}{2 \epsilon^{\prime}+1} \mathbf{M} \cdot \mathbf{E}_{0} .
\end{aligned}
$$

We now have constructed a system with periodic boundary conditions with an electrostatic energy expressed in terms of the charges and positions of the particles in the basic unit cell. This may be used as a contribution to the potential energy of a many-particle system in a molecular-dynamics or Monte Carlo simulation. The possibility of varying the potential through the choice of ellipsoidal shape, the outside dielectric constant $\epsilon^{\prime}$, and the applied electric field $\mathbf{E}_{0}$ permits selection of a suitable situation. Macroscopic arguments from thermodynamic fluctuation theory and Maxwell theory may be employed in this selection. For example, putting $\mathbf{E}_{0}=0$ and $\epsilon^{\prime}=\infty$, one finds from (2.7) that the Maxwell field vanishes and the last two terms in (4.5) and (4.7) drop out. In this case fluctuation theorems become particularly simple.

The first term in (4.5) is independent of the shape of the macroscopic sample. It may be expressed as a sum of pair interactions. Substituting (3.4) and using (3.2) and (4.1), we find

$$
\frac{1}{2} \sum_{j=1}^{N} e_{j} \phi_{c j}^{\prime}\left(\mathbf{R}_{j}, L\right)=\frac{1}{2 L} \sum_{j \neq k} e_{j} e_{k} w\left(\left(\mathbf{R}_{j}-\mathbf{R}_{k}\right) / L\right) .
$$

This shows that the crystal potential of the Wigner solid plays the role of pair interaction in periodic Coulomb systems.

\section{DIPOLAR SYSTEMS}

Dipolar systems may be treated as a special case of the ionic systems discussed in the preceding sections. In dipolar systems we deal with pairs of charges of opposite sign linked together at an infinitesimal distance. We slightly change notation and consider $N$ permanent dipole moments $\boldsymbol{\mu}_{1}, \ldots, \boldsymbol{\mu}_{N}$ situated at positions 
$\mathbf{R}_{1}, \ldots, \mathbf{R}_{N}$ in the basic cell. The total dipole moment of the basic cell becomes

$$
\mathbf{M}=\sum_{j=1}^{N} \mu_{j}
$$

We again consider the geometry $C\left(\Omega, L, \epsilon^{\prime}\right)$ for the case where $\Omega$ is an ellipsoid.

We define the crystal field acting on dipole moment $j$ in the basic cell by

$$
\begin{aligned}
\mathbf{E}_{c j}^{\prime}(\mathbf{r}, L)= & -\nabla \phi_{c}(\mathbf{r}, L) \\
& +\frac{\left(\mathbf{r}-\mathbf{R}_{j}\right)^{2} 1-3\left(\mathbf{r}-\mathbf{R}_{j}\right)\left(\mathbf{r}-\mathbf{R}_{j}\right)}{\left|\mathbf{r}-\mathbf{R}_{j}\right|^{5}} \cdot \boldsymbol{\mu}_{j} .
\end{aligned}
$$

Here we have subtracted a self-term for dipole moment $j$. The crystal potential $\phi_{c}(\mathbf{r}, L)$ is found in analogy to (3.1) from the Poisson equation

$$
\nabla^{2} \phi_{c}(\mathbf{r}, L)=4 \pi \sum_{k=1}^{N} \mu_{k} \cdot \nabla \delta\left(\mathbf{r}-\mathbf{R}_{k}\right), \quad r \in C(L)
$$

with periodic boundary conditions. In analogy to (3.4), the solution is given by

$$
\phi_{c}(\mathbf{r}, L)=-\frac{1}{L} \sum_{k=1}^{N} \mu_{k} \cdot \nabla w\left(\left(\mathbf{r}-\mathbf{R}_{k}\right) / L\right) .
$$

By comparison with (3.2) and (3.3) we therefore find that at $\mathbf{r}=\mathbf{R}_{j}$

$$
\mathbf{E}_{c j}^{\prime}\left(\mathbf{R}_{j}, L\right)=\frac{4 \pi}{3 L^{3}} \mu_{j}+\frac{1}{L} \sum_{k \neq j} \mu_{k} \cdot \nabla_{j} \nabla_{j} w\left(\left(\mathbf{R}_{j}-\mathbf{R}_{k}\right) / L\right)
$$

The first term is a self-term due to the periodic images of dipole moment $j$. We may rewrite (5.5) in the form

$$
\mathbf{E}_{c j}^{\prime}\left(\mathbf{R}_{j}, L\right)=\frac{4 \pi}{3 L^{3}} \mathbf{M}+\sum_{k \neq j} \mathrm{~T}_{w}\left(\mathbf{R}_{j}-\mathbf{R}_{k}\right) \cdot \boldsymbol{\mu}_{k},
$$

where the Wigner dipole tensor in the second term is given by

$$
\mathrm{T}_{w}(\mathbf{r})=L^{-1} \nabla \nabla w(\mathbf{r} / L)-\frac{4 \pi}{3 L^{3}} 1 .
$$

Here the last term cancels precisely against the second derivative of the second term in (3.2). The total field acting on dipole moment $j$ in the basic cell is given by

$$
\mathbf{E}_{j}^{\prime}\left(\Omega, L, \epsilon^{\prime}\right)=\mathbf{E}_{M}\left(\Omega, \epsilon^{\prime}\right)+\mathbf{E}_{c j}^{\prime}\left(\mathbf{R}_{j}, L\right),
$$

where the first term is given by (2.7). For $\epsilon^{\prime}=1$ and a spherical sample this takes the simple form

$$
\mathbf{E}_{j}^{\prime}(S, L, 1)=\mathbf{E}_{0}+\sum_{k \neq j} \mathbf{T}_{w}\left(\mathbf{R}_{j}-\mathbf{R}_{k}\right) \cdot \boldsymbol{\mu}_{k}
$$

In this special case there is no self-contribution.

For an ellipsoidal sample the electrostatic energy of the dipolar system is again a sum of equal contributions from the separate cubic cells, so that we may write the energy as in (4.4). The energy per cell is given by

$$
\begin{aligned}
U\left(\Omega, L, \epsilon^{\prime}\right)= & -\frac{1}{2} \sum_{j=1}^{N} \mu_{j} \cdot \mathbf{E}_{c j}^{\prime}\left(\mathbf{R}_{j}, L\right)-\frac{1}{2} \mathbf{M} \cdot \mathbf{E}_{M}^{P}\left(\Omega, \epsilon^{\prime}\right) \\
& -\mathbf{M} \cdot \mathbf{E}_{M}^{0}\left(\Omega, \epsilon^{\prime}\right)
\end{aligned}
$$

This expression for the energy is essentially identical to that derived earlier by one of us. ${ }^{8}$ In the previous article the acting crystal field was called the Ewald inner field. We abandon that nomenclature here, since the crystal field need not necessarily be calculated by Ewald's method. In the earlier article ${ }^{8}$ the crystal field was not given explicitly.

The first term in (5.10) is independent of the shape of the sample. It may be expressed as a sum of pair interactions in analogy to (4.8). We find here by use of (5.6)

$$
\sum_{j=1}^{N} \boldsymbol{\mu}_{j} \cdot \mathbf{E}_{c j}^{\prime}\left(\mathbf{R}_{j}, L\right)=\frac{4 \pi}{3 L^{3}} \mathbf{M}^{2}+\sum_{j \neq k} \mu_{j} \cdot \mathrm{T}_{w}\left(\mathbf{R}_{j}-\mathbf{R}_{k}\right) \cdot \mu_{k} .
$$

Substituting in (5.10) we find for the case of a sphere

$$
\begin{aligned}
U\left(S, L, \epsilon^{\prime}\right)= & -\frac{1}{2} \sum_{j \neq k} \boldsymbol{\mu}_{j} \cdot \mathrm{T}_{w}\left(\mathbf{R}_{j}-\mathbf{R}_{k}\right) \cdot \boldsymbol{\mu}_{k} \\
& -\frac{4 \pi}{3} \frac{\epsilon^{\prime}-1}{2 \epsilon^{\prime}+1} \frac{M^{2}}{V}-\frac{3 \epsilon^{\prime}}{2 \epsilon^{\prime}+1} \mathbf{M} \cdot \mathbf{E}_{0} .
\end{aligned}
$$

The first term may be evaluated once the basic potential $w(\mathbf{x})$ is known.

\section{WIGNER POTENTIAL}

In this section we study the basic potential function $w(\mathbf{x})$ in more detail. In Eq. (3.3) we have related $w(\mathbf{x})$ to the electrostatic potential of a Wigner solid. ${ }^{12}$ For lattice distance unity and for unit charge the two quantities are identical. We shall therefore simply call $w(\mathbf{x})$ the Wigner potential. In the literature $\mathrm{e}^{22,23}$ the potential is sometimes called the Ewald potential because it may be calculated by use of Ewald lattice sums. However, on the one hand Ewald's method is valid for more general models; on the other hand it is not the only method by which the potential may be calculated. We prefer the name Wigner potential, since it is uniquely associated with a Wigner crystal consisting of an electron lattice embedded in a uniform neutralizing background. ${ }^{12}$

It follows from (3.1) and (3.2) that in the basic unit cell the Wigner potential may be written

$$
\begin{array}{r}
w(\mathbf{r})=\frac{1}{r}+\frac{2 \pi}{3} r^{2}+\sum_{\substack{l>0 \\
m}}\left[\frac{4 \pi}{2 l+1}\right]^{1 / 2} A_{l m} r^{l} Y_{l m}(\theta, \varphi), \\
\mathbf{r} \in C(1) .
\end{array}
$$

Here the $Y_{l m}(\theta, \varphi)$ are spherical harmonics in the notation of Edmonds ${ }^{24}$ and we have chosen a convenient normalization for the superposition coefficients $A_{I m}$. It has been shown by de Wette and Nijboer ${ }^{15}$ that each coefficient $A_{l m}$ may be expressed as a lattice sum which may be evaluated by the Ewald method. 
In (6.1) the potential is expanded about the origin, where the unit charge is located. For our purposes it will be convenient to also consider expansions about different points in the unit cell. We denote coordinates defined with respect to a chosen point $\mathbf{R}_{1}$ by $\mathbf{r}_{1}=\mathbf{r}-\mathbf{R}_{1}$. We show in the Appendix that the expansion of $w(\mathbf{r})$ about a point $\mathbf{R}_{1}$ in the basic unit cell different from the origin is given by

$$
\begin{aligned}
w(\mathbf{r})=w\left(\mathbf{R}_{1}\right)+\frac{2 \pi}{3} r_{1}^{2}+ & \sum_{\substack{l>0 \\
m}} \\
\times & \left(\frac{4 \pi}{2 l+1}\right)^{1 / 2} \Psi_{l m}^{*}\left(\mathbf{R}_{1}\right) \\
& \times r_{1}^{l} Y_{l m}\left(\theta_{1}, \varphi_{1}\right)
\end{aligned}
$$

where $\left(r_{1}, \theta_{1}, \varphi_{1}\right)$ are the polar coordinates of $\mathbf{r}_{1}$ and $\Psi_{l m}(\mathbf{R})$ is given by the Ewald sum

$$
\begin{aligned}
\Psi_{l m}(\mathbf{R})=\left(\frac{4 \pi}{2 l+1}\right)^{1 / 2} \frac{1}{\Gamma\left(l+\frac{1}{2}\right)}( & \sum_{\mathbf{n}} \Gamma\left(l+\frac{1}{2}, \pi|\mathbf{n}-\mathbf{R}|^{2}\right)|\mathbf{n}-\mathbf{R}|^{-l-1} Y_{l m}\left(\theta_{\mathbf{n}-\mathbf{R}}, \varphi_{\mathbf{n}-\mathbf{R}}\right) \\
& \left.+i^{l} \pi^{l-1 / 2} \sum_{\mathbf{n}}^{\prime}|\mathbf{n}|^{l-2} \exp \left(2 \pi i \mathbf{n} \cdot \mathbf{R}-\pi|\mathbf{n}|^{2}\right) Y_{l m}\left(\theta_{\mathbf{n}}, \varphi_{\mathbf{n}}\right)\right) .
\end{aligned}
$$

The first sum is over the real-space lattice, and the second sum is over the reciprocal lattice with omission of the term $\mathbf{n}=0$. The coefficients $A_{l m}$ in (6.1) are given by

$$
A_{l m}=\left[\Psi_{l m}^{\prime}(0)\right]^{*},
$$

where the prime indicates that in the first sum in (6.3) the term $\mathbf{n}=0$ must be omitted as well.

Clearly the sum in (6.2) converges only in a sphere centered at $\mathbf{R}_{1}$ of radius $R_{1}$. We obtain a more rapidly converging sum by treating the potential from the nearest lattice points separately. We shall consider special points $\mathbf{R}_{1}$ in the basic unit cell about which we expand the potential. These are chosen such that many coefficients in the expansion vanish by symmetry. It suffices to consider the first octant of the basic unit cell, defined by the inequalities $0 \leq x \leq \frac{1}{2}, 0 \leq y \leq \frac{1}{2}$, and $0 \leq z \leq \frac{1}{2}$. We call the expansion (6.1) about the origin the $A$ expansion. Similarly there are three $B$ expansions centered about the three corners of the first octant $B_{1}=\left(\frac{1}{2}, 0,0\right)$, $B_{2}=\left(0, \frac{1}{2}, 0\right)$, and $B_{3}=\left(0,0, \frac{1}{2}\right)$. There are three $C$ expansions centered about the three corners $C_{1}=\left(0, \frac{1}{2}, \frac{1}{2}\right)$, $C_{2}=\left(\frac{1}{2}, 0, \frac{1}{2}\right)$, and $C_{3}=\left(\frac{1}{2}, \frac{1}{2}, 0\right)$. Finally there is one $D$ expansion centered about the corner $D=\left(\frac{1}{2}, \frac{1}{2}, \frac{1}{2}\right)$. We denote coordinate vectors relative to each of these seven corners by $\mathbf{r}^{\prime}$ and the positons of the charges by $\mathbf{n}^{\prime}$.

The $B$ expansions take the form

$$
\begin{aligned}
w(\mathbf{r})= & \sum_{\mathrm{NN}} \frac{1}{\left|\mathbf{r}^{\prime}-\mathbf{n}^{\prime}\right|}+\frac{2 \pi}{3} r^{\prime 2} \\
& +\sum_{l m}\left[\frac{4 \pi}{2 l+1}\right]^{1 / 2} B_{l m} r^{\prime l} Y_{l m}\left(\theta^{\prime}, \varphi^{\prime}\right), \quad \mathbf{r} \in C(1)
\end{aligned}
$$

where the first sum is over the lattice points nearest to the $B$ center. For example, for $B_{1}=\left(\frac{1}{2}, 0,0\right)$ the nearest lattice points are $(0,0,0)$ and $(1,0,0)$. The number of terms in the second sum may be minimized by choosing coordinates $x^{\prime}, y^{\prime}, z^{\prime}$ such that the potential is symmetric under inversion $\mathbf{r}^{\prime} \rightarrow-\mathbf{r}^{\prime}$ and the interchange $x^{\prime}, y^{\prime} \rightarrow y^{\prime}, x^{\prime}$. Then only even values of $l$ and values of $m$ which are multiples of four occur. For example, for $B_{1}=\left(\frac{1}{2}, 0,0\right)$ the $z^{\prime}$ axis is taken in the positive $x$ direction, the $x^{\prime}$ axis is taken in the $y$ direction, and the $y^{\prime}$ axis is taken in the $z$ direction.

The $C$ expansions take the form

$$
\begin{aligned}
w(\mathbf{r})= & \sum_{\mathrm{NN}} \frac{1}{\left|\mathbf{r}^{\prime}-\mathbf{n}^{\prime}\right|}+\frac{2 \pi}{3} r^{\prime 2} \\
& +\sum_{l m}\left(\frac{4 \pi}{2 l+1}\right)^{1 / 2} C_{l m} r^{\prime l} Y_{l m}\left(\theta^{\prime}, \varphi^{\prime}\right),
\end{aligned}
$$

$r \in C(1)$

(6.6)

where the first sum is over the lattice points nearest to the $C$ center. For example, for $C_{1}=\left(0, \frac{1}{2}, \frac{1}{2}\right)$ the nearest lattice points are $(0,0,0),(0,1,0),(0,0,1)$, and $(0,1,1)$. To minimize the number of terms in the second sum the $x^{\prime}$, $y^{\prime}$, and $z^{\prime}$ axes are chosen as above.

The $D$ expansion takes the form

$$
\begin{aligned}
w(\mathbf{r})= & \sum_{\mathrm{NN}} \frac{1}{\left|\mathbf{r}^{\prime}-\mathbf{n}^{\prime}\right|}+\frac{2 \pi}{3} r^{\prime 2} \\
& +\sum_{l m}\left[\frac{4 \pi}{2 l+1}\right]^{1 / 2} D_{l m} r^{\prime l} Y_{l m}\left(\theta^{\prime}, \varphi^{\prime}\right),
\end{aligned}
$$$$
r \in C(1)
$$

where the first sum is over the eight lattice sites nearest to $D$. In this case it is not necessary to rotate the axes.

It follows from $(6.5)-(6.7)$ that the coefficients $B_{00}$, $C_{00}$, and $D_{00}$ are given by

$$
\begin{aligned}
& B_{00}=w\left(B_{1}\right)-4, \\
& C_{00}=w\left(C_{1}\right)-4 \sqrt{2}, \\
& D_{00}=w(D)-16 / \sqrt{3},
\end{aligned}
$$

where $w\left(B_{1}\right), w\left(C_{1}\right)$, and $w(D)$ may be evaluated by an Ewald summation, as explained in Sec. VII. By comparison of (6.2) and (6.5) for the point $B_{3}$ we find that the coefficients $B_{l m}$ for $l>0$ are given by

$$
B_{l m}=\Psi_{l m}^{*}\left(B_{3}\right)-\left(\frac{4 \pi}{2 l+1}\right)^{1 / 2} \sum_{\mathrm{NN}}\left|\mathbf{n}^{\prime}\right|^{\cdots-1-1} Y_{l m}^{*}\left(\theta_{\mathrm{n}^{\prime}}, \varphi_{\mathrm{n}^{\prime}}\right) \text {. }
$$


More explicitly this reads

$$
B_{l m}=\Psi_{l m}^{*}\left(B_{3}\right)-2^{l+1}\left[1+(-1)^{l}\right] \delta_{m 0} .
$$

By comparison of (6.2) and (6.6) for the point $C_{3}$ we find for $l>0$

$C_{l m}=\Psi_{l m}^{*}\left(C_{3}\right)-\left[\frac{4 \pi}{2 l+1}\right]^{1 / 2} \sum_{\mathrm{NN}}\left|\mathbf{n}^{\prime}\right|^{-1-1} Y_{l m}^{*}\left(\theta_{\mathbf{n}^{\prime}}, \varphi_{\mathbf{n}^{\prime}}\right)$

Similarly we find by comparing (6.2) and (6.7) for the point $D$ for $l>0$

$D_{l m}=\Psi_{l m}^{*}(D)-\left(\frac{4 \pi}{2 l+1}\right)^{1 / 2} \sum_{\mathrm{NN}}\left|\mathbf{n}^{\prime}\right|^{-l-1} Y_{l m}^{*}\left(\theta_{\mathbf{n}^{\prime}}, \varphi_{\mathbf{n}^{\prime}}\right)$.

In the numerical evaluation of the expansions it is convenient to use the expressions for the spherical harmonics in terms of Cartesian coordinates. These may be obtained from the generating function given in Eq. $(\mathbf{H}-38)$ in the monograph by Normand. ${ }^{25}$ Thus we write the expansions (6.1), (6.5), (6.6), and (6.7) in the form

$w(\mathbf{r})=\sum_{\mathrm{NN}} \frac{1}{\left|\mathbf{r}^{\prime}-\mathbf{n}^{\prime}\right|}+\frac{2 \pi}{3} \boldsymbol{r}^{\prime 2}+\sum_{i, j, k \geq 0} a_{i j k} x^{\prime 2 i} y^{\prime 2 j} z^{\prime 2 k}$,

where in the case of (6.1) the first sum is to be replaced by $1 / r$. The second sum is a sum of solid spherical harmonics of all orders $l=2(i+j+k)$. Explicit values for the coefficients $a_{i j k}$ up to $l=14$ for the four types of corners of the first octant are listed on a diskette available from the authors upon request. In Table I we list the values of the coefficients $A_{l m}, \ldots, D_{l m}$ to an accuracy of ten decimal places up to $l=16$.

The advantage of our method is that the coefficients in the series expansions (6.13) need be evaluated only once. Using these coefficients one may evaluate the value of the Wigner potential $w(\mathbf{r})$ for any point in the first octant of the basic unit cell quickly and with high accuracy. For any given point one chooses the series expansion corresponding to the nearest corner. By construction this expansion converges rapidly. Any point in the remainder of the basic cell may be related to a point in the first octant by use of the cubic symmetry.

\section{COMPARISON WITH OTHER METHODS}

Several methods of calculation of the Wigner potential have been presented in the literature. In this section we compare these methods with the one proposed above. To introduce the other methods we note that an alternative expression for the Wigner potential may be found by solving Poisson's equation (3.1) by Fourier transformation. This yields

$$
w(\mathbf{r})=\mathfrak{J}+\frac{1}{\pi} \sum_{\mathbf{n}}^{\prime}|\mathbf{n}|^{-2} \exp (2 \pi i \mathbf{n} \cdot \mathbf{r})
$$

where the constant takes the value $\mathcal{J}=2.83729747948$. It was shown by Hasimoto $^{26}$ that the constant may be

\begin{tabular}{|c|c|c|c|c|c|}
\hline$l$ & $m$ & $A_{l m}$ & $\boldsymbol{B}_{l m}$ & $C_{l m}$ & $D_{l m}$ \\
\hline 0 & 0 & 0.0000000000 & -1.2586348255 & -3.4020783016 & -7.2022427976 \\
\hline 2 & 0 & 0.0000000000 & -0.9589795925 & 1.3229885794 & 0.0000000000 \\
\hline 4 & 0 & 3.1082266827 & -0.7040569101 & -0.3592213658 & 0.1718047780 \\
\hline 4 & 4 & 1.8575207277 & 1.1645026577 & -0.0505753286 & 0.1026729930 \\
\hline 6 & 0 & 0.5733292894 & 1.3493322222 & -0.8397029949 & 0.0073287913 \\
\hline 6 & 4 & -1.0726008854 & 0.9679735193 & -0.4873636202 & -0.0137109131 \\
\hline 8 & 0 & 3.2592930933 & -0.5968315208 & -0.0953548655 & -0.0713093808 \\
\hline 8 & 4 & 1.2256595032 & -0.8103790941 & -0.5165185628 & -0.0268159438 \\
\hline 8 & 8 & 1.8674436227 & 0.5915273908 & -0.0442486756 & -0.0408574021 \\
\hline 10 & 0 & 1.0092239881 & -0.0500926226 & 0.2083235107 & 0.0067412457 \\
\hline 10 & 4 & -1.0169576183 & 0.1282059875 & -0.2828084065 & -0.0067929035 \\
\hline 10 & 8 & -1.2104216743 & 0.8393417583 & 0.0461092921 & -0.0080851723 \\
\hline 12 & 0 & 2.8912541083 & 0.4030685989 & 0.1447586191 & -0.0042781638 \\
\hline 12 & 4 & 0.8916802213 & 0.3257794828 & -0.0033978318 & -0.0058279130 \\
\hline 12 & 8 & 1.0699741236 & -0.2001183693 & 0.0760578581 & 0.0049197960 \\
\hline 12 & 12 & 1.5887797580 & 0.1895278766 & -0.0068788937 & -0.0039122739 \\
\hline 14 & 0 & 1.1536367987 & -0.2963449157 & -0.0300769629 & 0.0014709727 \\
\hline 14 & 4 & -0.8483399039 & -0.3362747218 & 0.0979577582 & -0.0010816965 \\
\hline 14 & 8 & -0.9103423018 & -0.2676215265 & 0.1058551734 & -0.0011607541 \\
\hline 14 & 12 & -1.1053666131 & 0.4768208425 & -0.0009873432 & -0.0014094246 \\
\hline 16 & 0 & 2.7923562989 & 0.0527562980 & -0.0651264296 & 0.0006391092 \\
\hline 16 & 4 & 0.8007193579 & 0.1082954025 & 0.0558269227 & -0.0001235872 \\
\hline 16 & 8 & 0.8411315901 & 0.2571221571 & 0.0848074124 & 0.0003514668 \\
\hline 16 & 12 & 0.9479395892 & 0.1049531998 & -0.0085788400 & 0.0005290829 \\
\hline 16 & 16 & 1.5005847723 & 0.0765398444 & 0.0005460670 & 0.0002202715 \\
\hline
\end{tabular}

TABLE I. Table of coefficients $A_{l m}, B_{l m}, C_{l m}$, and $D_{l m}$ as defined in (6.1), (6.5), (6.6), and (6.7). 
evaluated from the Ewald sum

$$
\mathscr{I}=\frac{2 \alpha}{\sqrt{\pi}}+\frac{\pi}{\alpha^{2}}-\sum_{\mathbf{n}}^{\prime}\left(\frac{\operatorname{erfc}(\alpha|\mathbf{n}|)}{|\mathbf{n}|}+\frac{1}{\pi|\mathbf{n}|^{2}} e^{-\pi^{2} n^{2} / \alpha^{2}}\right)
$$

in which

$$
\operatorname{erfc}(x)=1-2 \pi^{-1 / 2} \int_{0}^{x} e^{-t^{2}} d t
$$

is the complementary error function. The parameter $\alpha$ may be chosen to optimize convergence of the lattice sums. With the choice $\alpha=\sqrt{\pi}$ the above expression was considered by Placzek et al. ${ }^{27}$ and by Nijboer. ${ }^{28}$ It has been shown by $\mathrm{Nijboer}^{28}$ that the constant $\mathcal{J}$ is related to the electrostatic energy of the Wigner lattice. The factor 1.760119 calculated for the simple cubic lattice by Coldwell-Horsfall and Maradudin ${ }^{29}$ is related to $\mathcal{J}$ by $1.760119=(3 / 4 \pi)^{1 / 3} \mathrm{~g}$.

By Ewald's method the Wigner potential may also be written as a sum of two contributions, the one a sum in real space and the other a sum in reciprocal space. In the notation of de Leeuw et $a l^{7}$

$$
w(\mathbf{r})=\psi(\mathbf{r})-\xi
$$

where both $\psi(\mathbf{r})$ and $\xi$ may be expressed in terms of Ewald lattice sums. The function $\psi(\mathbf{r})$ is given by

$$
\begin{aligned}
\psi(\mathbf{r})= & \sum_{\mathbf{n}} \frac{\operatorname{erfc}(\alpha|\mathbf{r}+\mathbf{n}|)}{|\mathbf{r}+\mathbf{n}|} \\
& +\frac{1}{\pi} \sum_{\mathbf{n}}^{\prime}|\mathbf{n}|^{-2} \exp \left(2 \pi i \mathbf{n} \cdot \mathbf{r}-\pi^{2}|\mathbf{n}|^{2} / \alpha^{2}\right) .
\end{aligned}
$$

The constant $\xi$ is given by

$$
\xi=\frac{\pi}{\alpha^{2}}-\mathfrak{J}
$$

This shows by comparison with (7.4) that $\psi(\mathbf{r})$ also depends on $\alpha$. We note that

$$
\xi=\lim _{r \rightarrow 0}[\psi(\mathbf{r})-1 / r] .
$$

The constants $B_{00}, C_{00}$, and $D_{00}$ in (6.8) may be evaluated from (7.4)-(7.6). The values are given in Table I.

We may write

$$
\psi(\mathbf{r})=\psi_{1}(r)+\psi_{2}(\mathbf{r})
$$

where the first term

$$
\psi_{1}(r)=\frac{\operatorname{erfc}(\alpha r)}{r}
$$

is isotropic and the second term

$$
\begin{aligned}
\psi_{2}(\mathbf{r})=\sum_{\mathbf{n} \neq 0}\left(\frac{\operatorname{erfc}(\alpha|\mathbf{r}+\mathbf{n}|)}{|\mathbf{r}+\mathbf{n}|}\right. \\
\left.+\frac{1}{\pi}|\mathbf{n}|^{-2} \exp \left(2 \pi i \mathbf{n} \cdot \mathbf{r}-\pi^{2}|\mathbf{n}|^{2} / \alpha^{2}\right)\right]
\end{aligned}
$$

is anisotropic. With the choice $\alpha=\sqrt{\pi}$ the above decom- position has been made by Brush et al. ${ }^{22}$ and by Hansen, ${ }^{23}$ apart from an additional -1 in (7.9). As noted before, an additional constant in the potential does not make any difference in the energy expressions (4.7) and (5.11).

For numerical purposes Hansen ${ }^{23}$ approximates $\psi_{2}(\mathbf{r})$ by an expansion in kubic harmonics with coefficients which are radial functions of $|\mathbf{r}|$ of a simple form chosen such as to reproduce $\psi_{2}(\mathbf{r})$ accurately at any point in the basic cell. With his choice of functions the relative error on the nonisotropic part of the potential is small (of the order of $1 \%$ or less) for all $r$. In absolute terms the potential is calculated accurately to three of four decimal digits. We have made a numerical comparison of Hansen's method with ours. We find that if we use harmonics up to $l=14$ we need about the same amount of computer time. At the center of the first octant, where convergence is worst, we then find $w(\mathbf{r})$ accurate to seven decimal digits. At most other points we find $w(\mathbf{r})$ accurate to 11 decimal digits. For example, at $\mathbf{r}=(0.3,0.3,0.3)$ we find $w(\mathbf{r})=2.362032963950$, Hansen's method yields $w(\mathbf{r})=2.364$, and the exact value calculated from (7.4) and (7.5) is $w(\mathbf{r})=2.362032963925$.

It may be that for some calculations involving ionic systems some inaccuracy in the potential may be tolerated. However, we have found that in dipolar systems, where the second derivatives of the potential are needed, the inaccuracy of the potential has disastrous consequences, especially at the edges of the basic cell. For dipolar systems an accurate calculation of the potential is an absolute necessity. By taking second derivatives one loses two decimal places of accuracy.

In the literature the expressions (7.4) and (7.5) have been used with a large but finite value of $\alpha$, such that in a calculation using the minimum image convention only one term of the first sum in (7.5) need be included. In numerical calculations values of $\alpha$ between 4 and 6 have been used. The advantage is that the remaining contribution to the total energy may be evaluated in Fourier space. This leads to a single particle sum for each wave vector, rather than a sum over pairs. We have found that even for the total energy our method is more accurate for the same amount of computer time. In other problems, where the actual values of the potential $w(\mathbf{r})$ or its derivatives are needed, the Ewald sums in (7.5) are far too time consuming.

The superiority of our method is due to the expansion in solid spherical harmonics about judiciously chosen points in the basic unit cell. This ensures that, even if the expansion is broken off and a finite sum is used, the Poisson equation for $w(\mathbf{r})$ is satisfied exactly at any point in the basic cell different from the origin. This is not the case for the Ewald sums in (7.5). In addition our method involves only simple algebraic manipulations and the numerical calculation may be easily vectorized.

\section{DISCUSSION}

We have developed a new method for calculating the effective Coulomb potential in periodic ionic and dipolar systems. The method allows fast and accurate calcula- 
tion of the interactions. We have tested the method in a calculation of the dielectric constant of nonpolar liquids. We shall report on this work separately.

Our method will be useful in computer simulations of ionic and dipolar systems involving a large number of particles in a unit cell, as well as in solid-state applications. As an example of an application in solid-state theory we evaluate the Madelung constant of an $\mathrm{NaCl}$ crystal. According to the prescriptions of Sec. II we must choose a unit cell of vanishing total charge. Thus, if $a$ is the lattice distance of the $\mathrm{NaCl}$ crystal we choose a cube with sides $2 a$ centered about the origin containing eight charges $\pm e$ of alternating sign at the points $\left( \pm \frac{1}{2} a, \pm \frac{1}{2} a, \pm \frac{1}{2} a\right)$. The dipole moment of the unit cell vanishes, so that the energy per cell follows from (4.4) and (4.7) with $\mathbf{M}=0$. The energy per particle is $\alpha_{M} e^{2} / a$, where $\alpha_{M}$ is the Madelung constant. From (4.7) we find

$$
\begin{aligned}
\alpha_{M} & =\frac{3}{2} w\left(\frac{1}{2}, \frac{1}{2}, 0\right)-\frac{3}{2} w\left(0,0, \frac{1}{2}\right)-\frac{1}{2} w\left(\frac{1}{2}, \frac{1}{2}, \frac{1}{2}\right) \\
& =-1.7475645946 .
\end{aligned}
$$

The separate contributions are, by use of (6.8) and Table I,

$$
\begin{aligned}
& w\left(0,0, \frac{1}{2}\right)=B_{00}+4=2.7413651745, \\
& w\left(\frac{1}{2}, \frac{1}{2}, 0\right)=C_{00}+4 \sqrt{2}=2.2547759479, \\
& w\left(\frac{1}{2}, \frac{1}{2}, \frac{1}{2}\right)=D_{00}+16 / \sqrt{3}=2.0353615094 .
\end{aligned}
$$

For many applications in solid-state theory the crystalline structure will differ from the simple cubic lattice studied in this article. The theory may be extended without difficulty to general Bravais lattices.

In computer simulations it is necessary to use an expression for the energy which takes account of the long range of the Coulomb interaction by including the Maxwell field for a large ellipsoidal sample, as first pointed out by de Leeuw et al. ${ }^{7}$ and by Felderhof. ${ }^{8}$ As discussed in Sec. IV, the possibility of including an applied electric field and a dielectric constant $\epsilon^{\prime}$ of the outer medium allows a flexibility which may be advantageously used. In selecting a suitable expression for the energy one may employ considerations from macroscopic Maxwell theory and thermodynamic fluctuation theory.

\section{ACKNOWLEDGMENTS}

We thank the Deutsche Forschungsgemeinschaft for financial support.

\section{APPENDIX}

In this appendix we discuss the derivation of the expansion (6.2) of the Wigner potential about an arbitrary point $\mathbf{R}_{1}$ in the basic unit cell different from the origin.

We begin by considering an arbitrary charge distribution $\rho(\mathbf{r})$ in vacuum and decompose its potential into a sum of two terms

$$
\phi(\mathbf{r})=\phi_{i}\left(\mathbf{r}, \mathbf{R}_{1}, a\right)+\phi_{0}\left(\mathbf{r}, \mathbf{R}_{1}, a\right) .
$$

Here

$$
\phi_{i}\left(\mathbf{r}, \mathbf{R}_{1}, a\right)=\int_{\left|\mathbf{r}^{\prime}-\mathbf{R}_{1}\right|<a} \frac{\rho\left(\mathbf{r}^{\prime}\right)}{\left|\mathbf{r}-\mathbf{r}^{\prime}\right|} d \mathbf{r}^{\prime}
$$

is the contribution from the charge density inside a sphere of radius $a$ about $\mathbf{R}_{1}$, and

$$
\phi_{0}\left(\mathbf{r}, \mathbf{R}_{1}, a\right)=\int_{\left|\mathbf{r}^{\prime}-\mathbf{R}_{1}\right|>a} \frac{\rho\left(\mathbf{r}^{\prime}\right)}{\left|\mathbf{r}-\mathbf{r}^{\prime}\right|} d \mathbf{r}^{\prime}
$$

is the contribution from the charge density outside the sphere. In the last expression we use the well-known expansion

$\frac{1}{\left|\mathbf{r}_{1}-\mathbf{r}_{1}^{\prime}\right|}=\sum_{l m} \frac{4 \pi}{2 l+1} \frac{r_{1<}^{l}}{r_{1>}^{l+1}} Y_{l m}\left(\theta_{1<}, \varphi_{1<}\right) Y_{l m}^{*}\left(\theta_{1>}, \varphi_{1>}\right)$,

where $\mathbf{r}_{1}=\mathbf{r}-\mathbf{R}_{1}, \mathbf{r}_{1}^{\prime}=\mathbf{r}^{\prime}-\mathbf{R}_{1}$, and $r_{1<}\left(r_{1>}\right)$ is the lesser (larger) of $r_{1}$ and $r_{1}^{\prime}$. Using this expansion in (A3) we find for $r_{1}<a$

$\phi_{0}\left(\mathbf{r}, \mathbf{R}_{1}, a\right)=\sum_{l m} \frac{4 \pi}{2 l+1} r_{1}^{l} Y_{l m}\left(\theta_{1}, \varphi_{1}\right) \Phi_{l m}\left(\mathbf{R}_{1}, a\right)$

with coefficients

$$
\Phi_{l m}\left(\mathbf{R}_{1}, a\right)=\int_{r_{1}^{\prime}>a} \frac{Y_{l m}^{*}\left(\theta_{1}^{\prime}, \varphi_{1}^{\prime}\right)}{r_{1}^{\prime l+1}} \rho\left(\mathbf{r}^{\prime}\right) d \mathbf{r}^{\prime} .
$$

The integral may be decomposed according to the method of Nijboer and de Wette. ${ }^{18}$ We write instead of (A6)

$\boldsymbol{\Phi}_{l m}\left(\mathbf{R}_{1}, a\right)=\Phi_{l m}^{(1)}\left(\mathbf{R}_{1}, a\right)+\Phi_{l m}^{(2)}\left(\mathbf{R}_{1}\right)+\Phi_{l m}^{(3)}\left(\mathbf{R}_{1}, a\right)$,

where the first term is given by the integral

$\Phi_{l m}^{(1)}\left(\mathbf{R}_{1}, a\right)=\int_{r_{1}^{\prime}>a} \frac{Y_{l m}^{*}\left(\theta_{1}^{\prime}, \varphi_{1}^{\prime}\right)}{r_{1}^{\prime l+1}} \mathscr{F}_{l}\left(\mathbf{r}_{1}^{\prime}\right) \rho\left(\mathbf{r}^{\prime}\right) d \mathbf{r}^{\prime}$,

the second term by

$\Phi_{l m}^{(2)}\left(\mathbf{R}_{1}\right)=\int \frac{Y_{l m}^{*}\left(\theta_{1}^{\prime}, \varphi_{1}^{\prime}\right)}{r_{1}^{\prime l+1}}\left[1-\mathcal{F}_{l}\left(\mathbf{r}_{1}^{\prime}\right)\right] \rho\left(\mathbf{r}^{\prime}\right) d \mathbf{r}^{\prime}$,

and the third term by

$\Phi_{l m}^{(3)}\left(\mathbf{R}_{1}, a\right)=-\int_{r_{1}^{\prime}<a} \frac{Y_{l m}^{*}\left(\theta_{1}^{\prime}, \varphi_{1}^{\prime}\right)}{r_{1}^{\prime l+1}}\left[1-\mathscr{F}_{l}\left(\mathbf{r}_{1}^{\prime}\right)\right] \rho\left(\mathbf{r}^{\prime}\right) d \mathbf{r}^{\prime}$.

(A10)

We leave $\Phi_{l m}^{(1)}$ and $\Phi_{l m}^{(3)}$ as they stand, but transform $\Phi_{l m}^{(2)}$ into an integral over reciprocal space with the aid of Parseval's theorem. We write

$$
G_{l m}(\mathbf{r})=\frac{Y_{l m}(\theta, \varphi)}{r^{l+1}}\left[1-\mathcal{F}_{l}(\mathbf{r})\right]
$$

and define the Fourier transforms

$$
\begin{aligned}
& \hat{\rho}(\mathbf{k})=\frac{1}{8 \pi^{3}} \int \rho(\mathbf{r}) e^{-i \mathbf{k} \cdot \mathbf{r}} d \mathbf{r} \\
& \hat{G}_{l m}(\mathbf{k})=\frac{1}{8 \pi^{3}} \int G_{l m}(\mathbf{r}) e^{-i \mathbf{k} \cdot \mathbf{r}} d \mathbf{r}
\end{aligned}
$$


According to Parseval's theorem

$$
\Phi_{l m}^{(2)}\left(\mathbf{R}_{1}\right)=8 \pi^{3} \int \hat{G}_{l m}^{*}(\mathbf{k}) \widehat{\rho}(\mathbf{k}) e^{i \mathbf{k} \cdot \mathbf{R}_{1}} d \mathbf{k}
$$

The advantage of the decomposition (A7) is that with a proper choice of the function $\mathcal{F}_{1}(\mathbf{r})$ the integrals in (A8) and (A13) may converge much more rapidly than the integral (A6).

We consider in particular the charge density of the Wigner crystal with lattice distance unity

$$
\rho(\mathbf{r})=\sum_{\mathbf{n}} \delta(\mathbf{r}-\mathbf{n})-1
$$

with the Fourier transform

$$
\hat{\rho}(\mathbf{k})=\sum_{\mathbf{n}}^{\prime} \delta(\mathbf{k}-2 \pi \mathbf{n}) .
$$

We also choose

$$
\mathscr{F}_{l}(\mathbf{r})=\Gamma\left(l+\frac{1}{2}, \pi r^{2}\right) / \Gamma\left(l+\frac{1}{2}\right),
$$

where $\Gamma(n, x)$ is the incomplete $\Gamma$ function. Furthermore we consider a point $\mathbf{R}_{1}$ in the basic unit cell and choose $a=R_{1}-\epsilon$, where $\epsilon$ is infinitesimal. Then the integral in (A8) yields for $l>0$



The integral in (A13) becomes

$$
\Phi_{l m}^{(2)}\left(\mathbf{R}_{1}\right)=8 \pi^{3} \sum_{\mathbf{n}}^{\prime} \hat{G}_{l m}^{*}(2 \pi \mathbf{n}) \exp \left(2 \pi i \mathbf{n} \cdot \mathbf{R}_{1}\right)
$$

From Eq. (A13) of Nijboer and de Wette ${ }^{18}$ we find

$$
\hat{G}_{l m}(\mathbf{k})=\frac{(-i)^{l}(k / 2)^{l-2}}{8 \pi^{3 / 2} \Gamma\left(l+\frac{1}{2}\right)} \exp \left(-k^{2} / 4 \pi\right) Y_{l m}\left(\theta_{\mathbf{k}}, \varphi_{\mathbf{k}}\right) .
$$

The integral in (A10) vanishes for $l>0$. Combining the above equations we find Eqs. (6.2) and (6.3). By considering in addition the potential $\phi_{i}\left(\mathbf{r}, \mathbf{R}_{1}, R_{1}\right)$ and the $l=0$ terms of the expansions we find

$$
\phi\left(\mathbf{R}_{1}\right)=w\left(\mathbf{R}_{1}\right)-\mathscr{J},
$$

where the value of $\mathscr{I}$ is given below (7.1). The result (A20) agrees with Hasimoto's ${ }^{26}$ expression (7.1).
*On leave of absence from Institute of Theoretical Physics, Warsaw University, Hoza 69, PL-00-681 Warsaw, Poland.

${ }^{1}$ B. J. Alder and E. L. Pollock, Annu. Rev. Phys. Chem. 32, 311 (1981).

${ }^{2}$ S. W. de Leeuw, J. W. Perram, and E. R. Smith, Annu. Rev. Phys. Chem. 37, 245 (1986).

${ }^{3}$ J.-P. Hansen, in Molecular-Dynamics Simulation of StatisticalMechanical Systems, edited by G. Ciccotti and W. G. Hoover (North-Holland, Amsterdam, 1986), p. 89.

${ }^{4}$ G. Bossis, C. Hesse-Bezot, and C. Brot, J. Chem. Phys. 80, 3399 (1984).

5J. A. Barker and R. O. Watts, Chem. Phys. Lett. 3, 144 (1969).

${ }^{6}$ E. L. Pollock and B. J. Alder, Physica A120, 1 (1980).

${ }^{7}$ S. W. de Leeuw, J. W. Perram, and E. R. Smith, Proc. R. Soc. London, Ser. A 373, 27 (1980); 373, 57 (1980).

${ }^{8}$ B. U. Felderhof, Physica A101, 275 (1980).

${ }^{9}$ S. W. de Leeuw, J. W. Perram, and E. R. Smith, Proc. R. Soc. London, Ser. A 388, 177 (1983).

${ }^{10} \mathrm{M}$. Neumann, O. Steinhauser, and G. Pawley, Mol. Phys. 52, 97 (1984).

${ }^{11}$ P. Linse and H. C. Andersen, J. Chem. Phys. 85, 3027 (1986).

${ }^{12}$ A. J. C. Ladd, Mol. Phys. 33, 1039 (1977); 36, 463 (1978).

${ }^{13}$ E. P. Wigner, Trans. Faraday Soc. 34, 678 (1938).

${ }^{14}$ L. D. Landau and E. M. Lifshitz, Electrodynamics of Continu- ous Media (Pergamon, Oxford, 1963), p. 44

${ }^{15}$ F. W. de Wette and B. R. A. Nijboer, Physica 24, 1105 (1958).

${ }^{16}$ B. U. Felderhof, Physica A130, 34 (1985).

${ }^{17}$ F. C. Von der Lage and H. A. Bethe, Phys. Rev. 71, 612 (1947); see also S. P. Altmann and A. P. Cracknell, Rev. Mod. Phys. 37, 19 (1965).

${ }^{18}$ B. R. A. Nijboer and F. W. de Wette, Physica 23, 309 (1957).

${ }^{19}$ F. W. de Wette, Phys. Rev. B 21, 3751 (1980).

${ }^{20} \mathrm{~J}$. Ihm and M. L. Cohen, Phys. Rev. B 21, 3754 (1980).

${ }^{21}$ B. R. A. Nijboer and Th.W. Ruijgrok, J. Stat. Phys. 53, 361 (1988).

${ }^{22}$ S. G. Brush, H. L. Sahlin, and E. Teller, J. Chem. Phys. 45, $2102(1966)$.

${ }^{23}$ J. P. Hansen, Phys. Rev. A 8, 3096 (1973).

${ }^{24}$ A. R. Edmonds, Angular Momentum in Quantum Mechanics (Princeton University Press, Princeton, NJ, 1974).

${ }^{25}$ J.-M. Normand, A Lie Group: Rotations in Quantum Mechanics (North-Holland, Amsterdam, 1980).

${ }^{26}$ H. Hasimoto, J. Fluid Mech. 5, 317 (1959).

${ }^{27}$ G. Placzek, B. R. A. Nijboer, and L. van Hove, Phys. Rev. 82, 392 (1951).

${ }^{28}$ B. R. A. Nijboer, Philips Res. Rep. 30, 74 (1975).

${ }^{29}$ R. A. Coldwell-Horsfall and A. A. Maradudin, J. Math. Phys. 1, 395 (1960) 